\title{
Selective Ion Isolation/Rejection Over a Broad Mass Range in the Quadrupole Ion Trap
}

\author{
Scott A. McLuckey, Douglas E. Goeringer, and Gary L. Glish \\ Analytical Chemistry Division, Oak Ridge National Laboratory, Oak Ridge, Tennessee, USA
}

\begin{abstract}
Techniques are presented for mass-selective ion manipulation over a wide mass range in a three-dimensional quadrupole. The methods use an auxiliary, low-amplitude radio-frequency signal applied to the endcap electrodes. This signal is either held at a single frequency as the fundamental radio-frequency trapping amplitude is ramped or swept over a frequency range while the fundamental radio-frequency trapping amplitude is held at a fixed level. Ion isolation and ejection are demonstrated for ions formed within the ion trap using electron ionization and for ions injected into the ion trap formed either by an air-sustained glow discharge or by electrospray. Mass-selective ion ejection is used to reduce matrix-ion-induced space charge during ion injection, thereby producing signal enhancement for the detection of 2,4,6-trinitrotoluene in air. Mass-selective isolation of ions with mass-to-charge ratios above the normal operating range $(\mathrm{m} / \mathrm{z} 650)$ for the ion trap is also demonstrated after injection of myoglobin ions formed via electrospray. (J Am Soc Mass Spectrom 1991, 2, 11-21)
\end{abstract}

$\mathrm{U}$ SE of the three-dimensional quadrupole (or quadrupole ion trap) has increased rapidly since the advent of the mass-selective instability technique of Stafford and Kelley and co-workers for obtaining mass spectra $[1,2]$ and the demonstration of improved mass resolution and sensitivity afforded by the use of helium (He) as a bath gas [3]. With the mass-selective instability approach, the ion trap can serve as a mass spectrometer simply by scanning the amplitude of the fundamental radiofrequency ( $\mathrm{rf}$ ) voltage that is applied to the ring electrode. The capabilities of the ion trap are further expanded when additional signals are applied to the ion-trap electrodes. For example, the application of direct-current (dc) voltage to either the endcaps or ring electrode (in conjunction with the if voltage applied to the ring electrode) allows for the selective isolation of ions with specific mass-to-charge ratios, that is, mass-selective storage, in analogy to the closely related quadrupole mass filter $[4,5])$. The application of a separate, low-amplitude (0.1-30 V p-p) rf signal to the end caps of the ion trap has also been shown to be useful for a variety of applications. The purpose of this signal is to kinetically excite ions in the axial dimension, that is, the endcap-to-endcap dimension. Kinetic excitation of the ions in this manner can be used either to cause ions to undergo increasingly

Address reprint requests to Scott A. McLuckey, Analytical Chemistry Division, Oak Ridge National Laboratory, Oak Ridge TN, 37831-6365. energetic collisions with the $\mathrm{He}$ bath gas while remaining in the trapping field or to throw ions from the trapping ficld. The former approach provides a convenient means of effecting collision-induced dissociation (CID) [6] in the ion trap. Coupling ion isolation with CID gives the capability for performing tandem mass spectrometry (MS/MS) [6-8]. The latter approach, which is referred to herein as resonance ejection, has also been found to be useful and is the main focus of this communication. For example, mass resolution of the ion trap is improved by a special form of kinetic excitation called axial modulation [9]. Techniques based on resonance ejection also enable the mass range of the ion trap to extend as high as $\mathrm{m} / \mathrm{z}$ 44,000 [10-12].

The purpose of this article is to present some novel resonance ejection techniques for mass-selective ion rejection. We restrict the discussion to dipolar excitation-cases in which the resonance ejection signals applied to the endcaps are of equal frequency and amplitude but are $180^{\circ}$ out of phase-and to cases where only one frequency is applied to the endcaps at any given time. We have already found several of the new techniques to be particularly useful in approaching problems faced in our laboratory, and we illustrate them with relevant examples. Other techniques that should also find practical uses are illustrated with ions derived from electron ionization (EI) of perfluorotributylamine, a commun mass calibration compound. The uses described herein include, for example, the selective rejection of a wide mass range dur- 


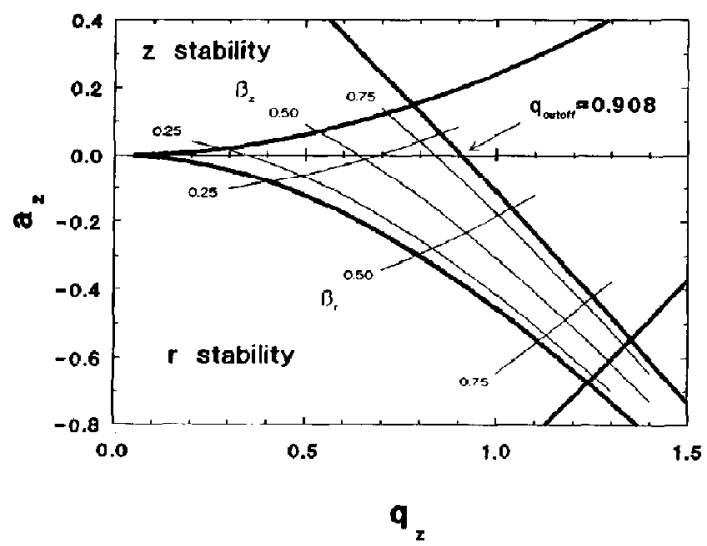

Figure 1. Stability diagram for the quadrupole ion trap.

ing storage of both higher and lower mass ions, the isolation of an intermediate range of masses, the discrete isolation of ions having widely spaced $\mathrm{m} / \mathrm{z}$ values, the isolation of ions with mass-to-charge ratios higher than the nominal mass range of the ion trap, and the use of resonance ejection during ion accumulation to reduce deleterious effects due to space charge.

\section{Theory}

\section{Basic Operation}

Fundamentals of ion trap conlainment theory are detailed elsewhere [13]. Briefly, the equations of motion for an ion in a three-dimensional quadrupole field can be written in a final form conforming with the Mathieu equation, the general form of which is characterized by the parameters $a_{u}$ and $q_{u}$. The subscript $u$ refers to either the axial $(z)$ or radial $(r)$ direction. These parameters are related to the if amplitude $(V)$, dc amplitude $(U)$, ring electrode radius $\left(r_{0}\right)$, angular radiofrequency $(\Omega)$, and mass-to-charge ratio $(\mathrm{m} / \mathrm{z})$ by eqs 1 and 2 .

$$
\begin{aligned}
& a_{z}=-2 a_{r}=-8 e U /\left(m r_{0}^{2} \Omega^{2}\right) \\
& q_{z}=-2 q_{r}=-4 e V /\left(m r_{0}^{2} \Omega_{2}\right)
\end{aligned}
$$

Graphical representations of stable solutions for the equations of motion in terms of the $a_{k}$ and $q_{k}$ parameters are called stability diagrams. The intersection of the loci of $a_{u}$ and $q_{u}$ points corresponds to conditions for simultaneously stable ion trajectories in both the axial and radial directions within the device. One such region of stability that is pertinent to this discussion is shown in Figure 1. The region is bounded by iso- $\beta_{u}$ lines having values of 0 and 1 ; other lines, for which $0<\beta_{u}<1$, fall within the stability diagram. $\beta_{u}$ is a complex function of $a_{u}$ and $q_{u}$. The trajectories of trapped ions are complex, but their motions are characterized by so-called secular frequencies, $\omega_{u r}$ with a superimposed micromotion frequency $\Omega$ [14]. The iso- $\beta_{u}$ lines indicate loci of $a_{u}$ and $q_{u}$ values for which all ions have the same secular frequencies. Of particular relevance to this discussion is the fundamental secular frequency in the axial dimension, $\omega_{0, z}$, which is related to $\beta_{z}$ by the equation

$$
\omega_{0, z}=2 \pi f_{0, z}=\beta_{z} \Omega / 2
$$

The basis for axial modulation and resonance ejection, as they are most commonly employed, is the kinetic excitation of ions having a fundamental axial secular frequency that matches the frequency applied to the endcaps. When the frequency of this relatively small (0.1-30 V p-p) signal becomes resonant with $f_{0, z}$ for a selected $\mathrm{m} / z$ value, ions characterized by that $\mathrm{m} / \mathrm{z}$ value absorb power and increase the amplitude of their oscillations [15].

\section{Current Isolation / Ejection Methods}

Several methods are currently used for isolation and ejection of ions stored in the ion trap: (1) mass-selective instability, (2) combination rf-dc, and (3) combination rf-resonance ejection. Each is briefly described below.

Mass-Selective instability. This method is normally used for generating mass spectra of trapped ions by sequential ejection from low to high mass [1]. The ion trap is operated in rf-only mode $\left(a_{u}=0\right)$, so that the locus of all working points is mapped onto the $q_{u}$ axis of the stability diagram. Ions with $q_{z}$ values below $q_{\text {cutoff }}=0.908\left(\beta_{z}=1\right)$ have stable trajectories and are stored. Ramping the magnitude of the if potential causes $q_{z}$ values for trapped ions to increase. As ions attain $q_{z}$ values of $q_{\text {cutoff }}$ they become unstable in the axial direction and are ejected from the trap. Massselective instability is also used for high-pass mass filtering of trapped ions. Because the $m / z$ value corresponding to $q_{\text {cutoff }}$ is determined by the rf trapping potential, ions below a selected $m / z$ are removed by adjusting the if amplitude to a value calculated from eq 2.

Combination $r f-d c$. When rf and dc potentials are applied simultaneously to the electrodes of the ion trap, such that the ratio for $U / V$ is maintained at a constant value, the locus of $m / z$ values lies along a working line that passes from the origin of the stability diagram through two of its boundaries. Adjustment of the $U / V$ ratio so that the working line crosses the boundaries near an apex permits mass-selective ion isolation. The specific $m / z$ value for trapped ions is determined by the rf and dc amplitudes and thus their $q_{u}$ and $a_{u}$ values. The combination of $\mathrm{rf}$ and $\mathrm{dc}$ is used primarily to isolate ions of a single mass-tocharge ratio. However, Todd et al. [16] also demonstrated that trapped ions can be low-pass mass-filtered 
by using a variation of this technique in which the $\mathrm{rf}$ amplitude is ramped from high to low amplitude while the dc level is fixed. Thus, ions above a selected $m / z$ value are ejected at the $\beta_{z}=0$ boundary. It is important to note that only an individual or continuous range of $m / z$ values can be isolated by means of these methods. Ions having discrete, widely spaced $m / z$ values cannot be simultaneously isolated with these techniques.

Combination $r$-resonance ejection. Ion ejection is offected by both mass-selective instability and resonance ejection in the technique demonstrated by Kelley et al. [17] fur isolation of a single ion species. A resonance ejection frequency equal to $f_{0, z}$ for an ion of $m / z$ value slightly greater than that of the targeted ion is applied while the rf amplitude is ramped. Ions below the selected $m / z$ value are ejected as their $q_{2}$ values exceed $q_{\text {cutoff }}\left(\beta_{z}=1\right)$, and concurrently ions above the desired $m / z$ value are sequentially removed as they become resonant with the resonance ejection frequency.

\section{Wide-Mass-Range Isolation / Ejection Methods}

The methods for mass-selective ion isolation/ejection reported in this article have the flexibility for targeting a single mass-to-rharge ratio, a wide $m / z$ range, or multiple, discrete, widely spaced $m / z$ values. The techniques are based on using either single-frequency resonance ejection/rf amplitude ramp or sweptfrequency resonance ejection/fixed if amplitude operation. The fundamental operation of each is described below.

Single-frequency resonance ejection/ $r f$ ramp. This method is an extension of the combination rf-resonance ejection technique of Kelley et al. described above for isolation of ions of a single $m / z$ value. Figure 2 shows an operational scenario in which $\mathrm{m} / \mathrm{z}$ ranges $25-50$ and $100-200$, indicated by the horizontal dashed lines, are simultaneously ejected while the $m / z$ ranges $50-100$ and $>200$ are retained in the trap. Because no dc potential is used, only the $q_{z}$ axis of the stability diagram is shown. Mass-to-charge ratios, calculated from eq 2 with $r_{0}=1.0 \mathrm{~cm}$ and $\Omega / 2 \pi$ $=1.1 \mathrm{MHz}$, are plotted versus $q_{z}$ for each of two $\mathrm{rf}$ amplitudes. The initial $\mathrm{rf}$ potential is $281 \mathrm{~V}(0-\mathrm{p})$, which corresponds to a high-pass $\mathrm{m} / \mathrm{z}_{\text {cutoff }}$ of 25 $\left(q_{z}=0.908\right)$. The $\mathrm{rf}$ amplitude is then ramped to $562 \mathrm{~V}$ (high-pass $m / z_{\text {cutoff }}-50$ ), as indicated by the vertical arrow at the right, causing the $m / z$ range $25-50$ to be sequentially removed as their $q_{z}$ values exceed 0.908 . Concurrent with the rf ramp, a resonance ejection signal is applied at a frequency $(89.2 \mathrm{kHz})$ corresponding to $q_{z}=0.227 \quad\left(\beta_{z}=0.162\right)$. The $\mathrm{m} / z$ values $100-200$, indicated by the left vertical arrow, sequentially come into resonance with the resonance ejection frequency and are also ejected. Other mass ranges can

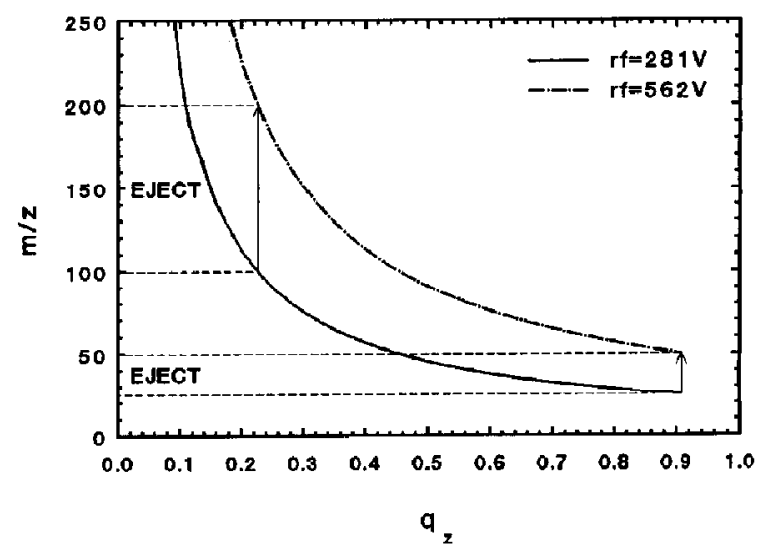

Figure 2. Diagram for selective ejection of ions at $m / z$ 100-200 via the simultaneous $\mathrm{rf}$-ramp/fixed-frequency resonance ejection technique.

be similarly targeted by proper selection of rf potential ranges and resonance ejection frequencies.

Swept-frequency resonance ejection / fixed rf. A selected mass range can also be ejected by sweeping the resonance ejection frequency while maintaining a fixed $\mathrm{rf}$ trapping level. An operational diagram for ejection of mass range $100-200$ via this technique is shown in Figure 3. Calculations for the plot were made with $r_{0}=1.0 \mathrm{~cm}, \Omega / 2 \pi=1.1 \mathrm{MHz}$, and the $\mathrm{rf}$ trapping potential fixed at $281 \mathrm{~V}$ (high-pass $m / z_{\text {cutoff }}=25$ ). Because $\beta_{z}$ and therefore $f_{0, z}$ (eq 3) are functions of $q_{z}$, secular frequency as well as $m / z$ are plotted versus $q_{z}$. Note that $f_{0, z}$ varies inversely with mass-tocharge ratio. The secular frequencies $89.2 \mathrm{kHz}$ and $44.2 \mathrm{kHz}$, corresponding to $m / z 100$ and 200 , respectively, are indicated by the dashed arrows running from the $m / z$ axis to the secular frequency axis. Ions with $\mathrm{m} / z$ values between 100 and 200 have secular frequencies between those indicated on the plot. Con-

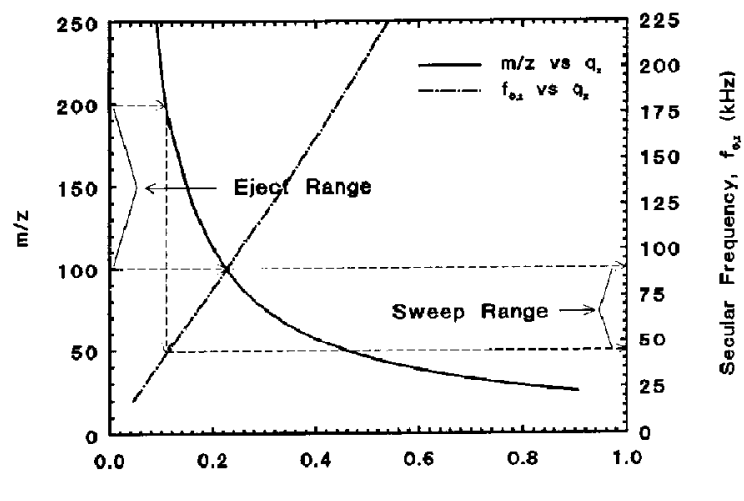

$\mathbf{q}_{x}$

Figure 3. Diagram for selective ejection of ions at $m / z$ 100-200 via the simultaneous swept-frequency resonance ejection/frxed-amplitude if technique. 
sequently, ions within the mass range $m / z$ 100-200 are ejected by sweeping the resonance ejection frequency between the frequency limits indicated in the diagram. Clearly, proper selection of frequency-sweep limits for the resonance ejection signal can allow other designated mass ranges to be similarly ejected.

Transform techniques. Syka and Fies [18] reported the application of Fourier transform methods to the mass-selective detection of ions in the quadrupole ion trap. Transient excitation, effected by application of an if pulse to the endcap electrodes, was used to impart coherent motion to trapped ions of the same $m / z$ value. Fourier transform analysis of the resultant ion image current signal transient revealed a frequency spectrum related to the $\mathrm{m} / \mathrm{z}$ values of the stored ions. Although these results suggest that a similar technique could be used to eject ions over a predetermined mass range, we have limited this study to the two less sophisticated isolation/ejection techniques described directly above.

\section{Experimental Section}

\section{Ion Trap Mass Spectrometer}

A Finnigan-MAT (San Jose, CA) ion trap mass spectrometer (ITMS) $\left(r_{0}=1.0 \mathrm{~cm}, \Omega / 2 \pi=1.1 \mathrm{MHz}\right)$ was used for experiments in which ions were formed via EI. Ion injection experiments were performed on a modified version of the ITMS adapted for use with external ion sources. The previously described instrument [19], as used for atmospheric sampling, consists of an atmospheric sampling glow discharge ionization (ASGDI) source [20] interfaced to a quadrupole ion trap via a three-element lens. The central element of the lens is electronically pulsed to gate ions into the ion trap through an endcap electrode. This basic interface, sans glow discharge, was also used for coupling an electrospray ionization (ESI) source to the modified ion trap [12]. A syringe pump (Harvard Apparatus, Inc., Cambridge, MA) was used to deliver a solution of horse skeletal muscle myoglobin at a rate of 1.0 $\mu \mathrm{L} / \mathrm{min}$ through a dome-tipped needle $(120 \mu \mathrm{m}$ i.d.) held at $3.3 \mathrm{kV}$. The needle tip was located $5-10 \mathrm{~mm}$ from the inlet to the intermediate pressure $(0.3$ torr) interface region. The analyzer regions of the instruments were maintained at $1-3$ mtorr of $\mathrm{He}$ for all experiments.

\section{Control Electronics}

Figure 4 is a block diagram of the electronics used for these experiments. Basic ion trap timing and control signals were supplied by the standard Finnigan-MAT ITMS electronics module. The ion/electron gate and axial modulation output signals were available on external BNC connectors. The trig output and gate control input signals were accessed via direct connection to



Figure 4. Diagram of electronic setup for resonance ejection experiments.

the appropriate integrated circuits on the scan acquisition processor circuit board. The ion / electron gate output was used to gate either electrons for EI or ions from an external source, as detailed above, into the ion trap. Certain experiments described below required external control of electron or ion gating. In those situations a pulse/delay generator, triggered from the ITMS trig output, provided the gate control input signal to the ITMS electronics. The ITMS internal frequency synthesizer was used for those experiments requiring only single-frequency resonance ejection. Swept-frequency resonance ejection was provided by an external function generator (Model 178, Wavetek, San Diego, CA); the delay output from the pulse/delay generator was used to gate the resonance ejection signal via amplitude modulation of the function generator output. Duration and delay for the gate control and modulation control signals were manually adjusted by observation on an oscilloscope. Amplitude, frequency, and sweep range of the sine wave signal frum the function generator were also manually set. The resonance ejection signal was fed into the ITMS "balun box," which produced two identical output signals phase-shifted by $180^{\circ}$. These signals were then applied to the endcaps. The resonance ejection signal was amplified when necessary by a fixed-gain (50 dB) rf amplifier (ENI Model 2100L, Rochester, NY) before entering the balun box.

\section{Data Acquisition}

Ion trap scan functions were developed using Finnigan-MAT version 4.1 software. Timing diagrams for two typical scan functions are shown in Figure 5. Figure 5a depicts signal timing for ion isolation/ejection via the single-frequency resonance ejection/rf ramp technique represented in Figure 2; the sweptfrequency resonance ejection/fixed if method represented in Figure 3 is diagramed in Figure 5b. The trigger output signal initiating each scan is followed 

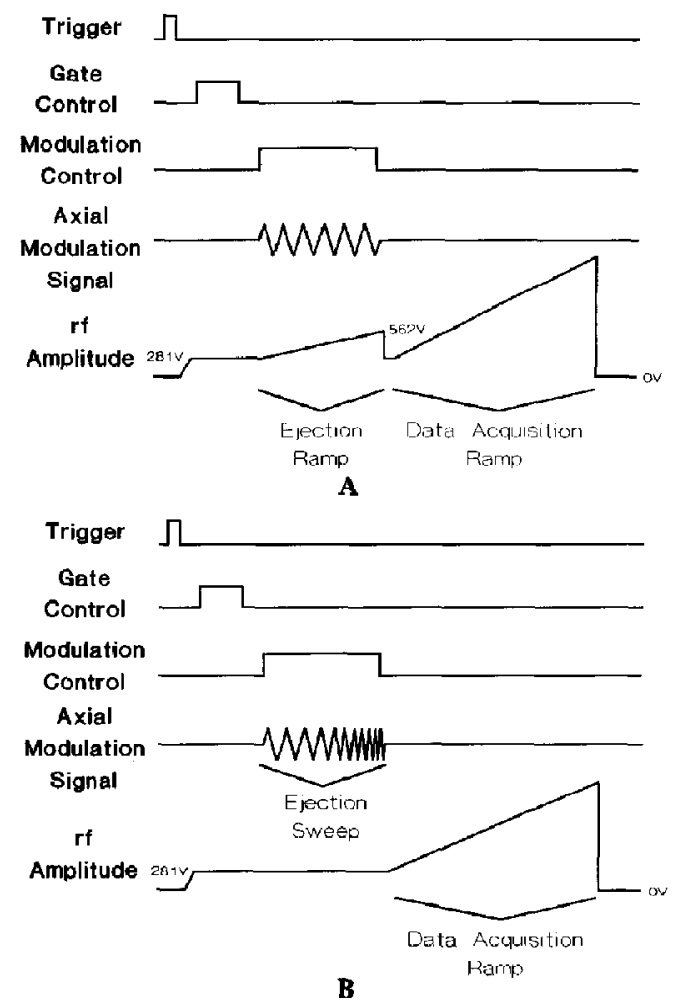

Figure 5. Scan functions for simultaneous (a) rf-ramp/fixedfrequency resonance ejection and (b) swept-frequency resonance ejection/fixed-amplitude if subsequent to ion accumulation in the ion trap.

by the gate control pulse, which effects ion formation or injection. Ion isolation/ejection is subsequent to ion accumulation in each scenario; situations in which isolation/ejection is concurrent with ion accumulation would indicate the gate control overlapped in time with the modulation control and/or the rf ramp. However, limitations in the standard ITMS software preclude ramping the if amplitude during the EI gating period. Therefore, the external pulse/delay generator described above was used to produce a gate control pulse during concurrent operation. Following ion formation or injection, the resonance ejection signal, either fixed or swept frequency, is gated on by the modulation control pulse while the if amplitude is ramped or held fixed. Subsequently, mass spectral data are acquired during the data acquisition ramp.

\section{Samples}

Perfluorotributylamine was supplied with the ITMS for mass calibration purposes. Myoglobin was commercially obtained and was dissolved at a concentration of $5 \mathrm{pmol} / \mu \mathrm{L}$ in a solvent mixture composed of high-performance liquid chromatography-grade water, methanol, and glacial acetic acid in relative approximate proportions of $20 \%, 75 \%$, and $5 \%$, respec- tively, by volume. Vapors of 2,4,6-trinitrotoluene (TNT) were sampled directly into the ASGDI source from the headspace in a 25-mL vial containing about 1 $\mathrm{mg}$ of TNT crystals held at room temperature. The concentration of TNT present in the air sampled into the ASGDI source under these circumstances is a few parts per billion by volume.

\section{Results and Discussion}

The results presented here were selected to demonstrate the capabilities and advantages of resonance ejection techniques for ion manipulation over a wide mass range. Two general types of situations are illustrated: one in which mass-selective ejection of unwanted ions is the primary objective, and another in which mass-selective isolation is emphasized. (Both, of course, occur in each experiment and are distinguished only according to one's point of view.) For each situation, results are presented using singlefrequency resonance ejection/rf ramp and frequencysweep resonance ejection/fixed-rf methods subsequent to ion formation or injection. Specificity for mass-selective ion ejection is also demonstrated for the latter technique. Advantages of the techniques are then presented for analytical applications. More specifically, reduction of space-charge effects associated with accumulation of matrix ions during ion injection and the ability to isolate ions with $\mathrm{m} / \mathrm{z}$ values greater than the normal mass range of the ITMS ( $m / z 650$ ) are demonstrated.

Mass spectra of positive ions formed via EI of PFTBA, a compound typically used for mass calibration in the ion trap, and negative ions from ASGDI of TNT were chosen to illustrate the general ion-manipulation capabilities of the techniques. Figure 6a shows a typical EI mass spectrum of PFTBA, and a representative ASGDI mass spectrum of TNT vapors in air [19] is presented in Figure $6 \mathrm{~b}$. The signal at $m / z 227$ in Figure $6 \mathrm{~b}$ corresponds to the molecular anion of TNT. Fragment anions at $m / z 210$ and 197 result from the loss of $\mathrm{OH} \cdot$ and $\mathrm{NO} \cdot$, respectively. The segment of the anion spectrum below $m / z 150$ is dominated by peaks typically observed from ambient air subjected to ASGDI. By far the major ions in the spectrum are $\mathrm{NO}_{2}^{-}(m / z 46), \mathrm{O}_{3}^{-}(m / z 48), \mathrm{CO}_{3}^{-}(m / z 60), \mathrm{HCO}_{3}^{-}$ $(m / z 61)$, and $\mathrm{NO}_{3}^{-}(m / z 62)$, whereas the total number of TNT-related ions ( $m / z 197,210$, and 227 ) constitute less than $1 \%$ of the ions in the mass spectrum.

\section{Mass-Selective Ejection}

Figure 7 shows PFTBA mass spectra obtained after ejection of ions within the targeted range $m / z$ 100-200 via the wide-mass-range ejection methods described above. A scan function similar to that in Figure 5a employing single-frequency resonance ejection/rf ramp operation was used to obtain the spectrum in 


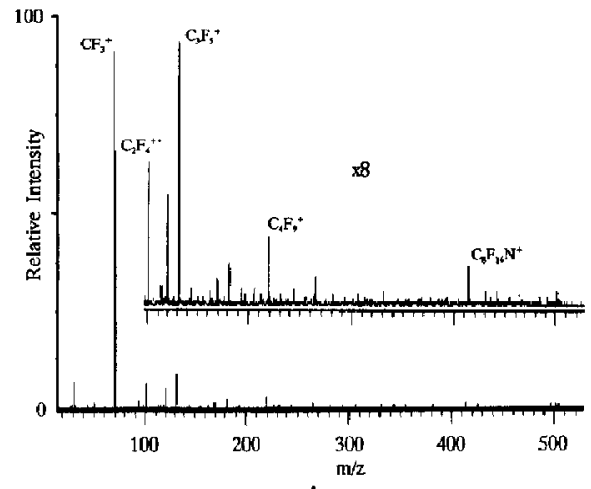

A

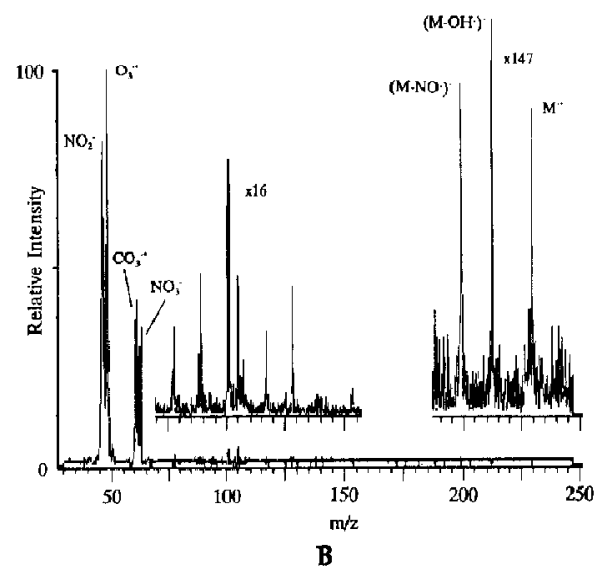

Figure 6. Typical ion trap mass spectra for (a) EI of PFTBA vapor and (b) injection of anions from air-sustained glow discharge ionization of TNT.

Figure $7 \mathrm{a}$, hence the process for ion ejection is described by the scenario diagramed in Figure 2. A $5-\mathrm{ms}, 6-\mathrm{V}(\mathrm{p}-\mathrm{p})$ resonance ejection signal from the ITMS electronics was applied to the endcaps as the if level on the ring electrode was ramped. The if amplitude limits and resonance ejection frequency were adjusted to correspond with the $m / z$ values $25-50$ and 100 , respectively. The spectrum in Figure $7 \mathrm{~b}$ was acquired with a swept-frequency resonance ejection/fixed rf scan function similar to that in Figure $5 b$, thus effecting ion ejection in the manner depicted in Figure 3. A 1-V (p-p) resonance ejection signal was applied for $30 \mathrm{~ms}$ as its frequency was swept over the range $44-89 \mathrm{kHz}$ at $4.5 \mathrm{kHz} / \mathrm{ms}$; the rf amplitude was set corresponding to a $q_{\text {cutoff }}$ for an ion of $m / z 25$. Note that these experimental scan parameters correspond with those plotted in Figures 2 and 3 . In each case the ions within the targeted mass range were sequentially ejected as they became resonant with the resonance ejection signal. Incomplete removal of ions at $m / z 100$ (low limit of targeted $m / z$ range) in Figure $7 \mathrm{~b}$ was due to deviations in the actual performance of the ion trap as compared with theoretical operation, that is, the experimental resonance fre-

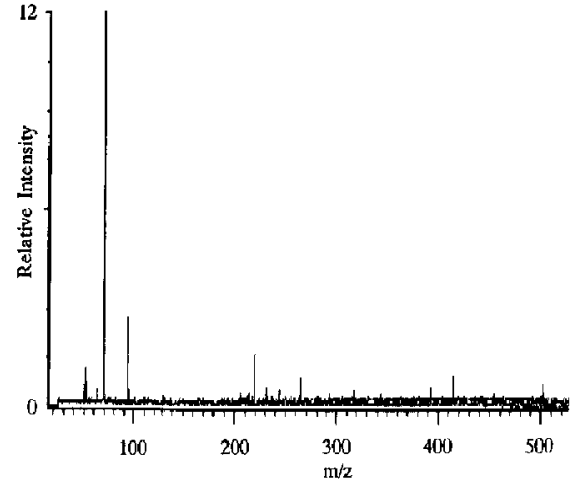

A

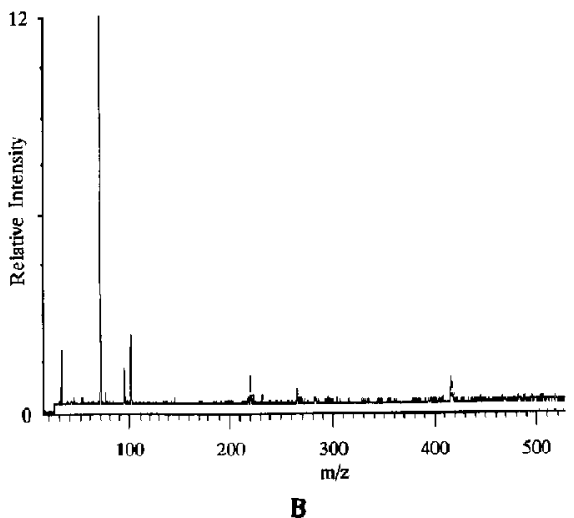

Figure 7. PFTBA mass spectra following ejection of ions at $m / z$ 100-200 via (a) $\mathrm{rf}$-ramp/hxed-frequency resonance ejection and (b) swept-frequency resonance ejection/fixed-amplitude if techniques.

quency range for ions of $m / z 100-200$ did not exactly match the calculated resonance ejection sweep range plotted in Figure 3. However, complete ion ejection of $m / z 100-200$ can be accomplished by empirical adjustment of the resonance ejection swecp range. $\Lambda \mathrm{l}$ though it is not readily apparent because of low ion signal initially present at $m / z 25-50$, the rf ramp method also ejected $m / z$ 25-50, whereas the swept frequency technique affected only ions in the range $m / z 100-200$. This effect can be used to advantage for wide mass range ion isolation, as will be apparent later.

The mass-selectivity resolution of the swept-frequency resonance ejection/fixed rf method was also investigated. A region of the ASGDI mass spectrum of TNT prior to ejection of ions between $m / z 46$ and 62 is shown in Figure 8a. Figure 8b shows the mass spectrum after a $3-\mathrm{V}(\mathrm{p}-\mathrm{p})$ resonance ejection signal was applied for $30 \mathrm{~ms}$; its frequency was swept over the range $191-271 \mathrm{kHz}$ at $8 \mathrm{kHz} / \mathrm{ms}$. As indicated in the spectrum, ions at $m / z 48,50,60$, and 61 were selectively removed while those at $\mathrm{m} / \mathrm{z} 46$ and 62 remained trapped. Increasing the resonance ejection amplitude produced a decrease in signal at $m / z 46$ 


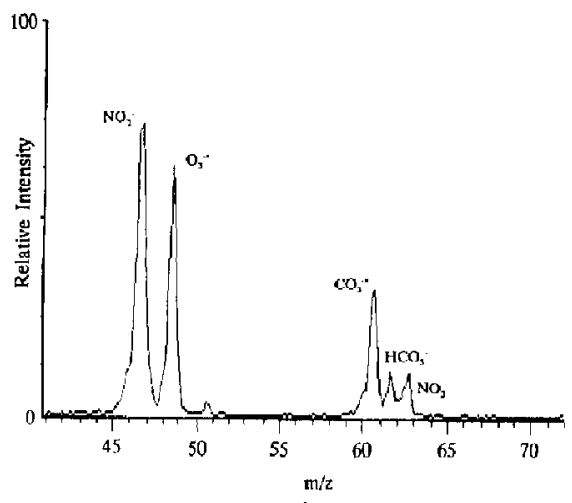

A

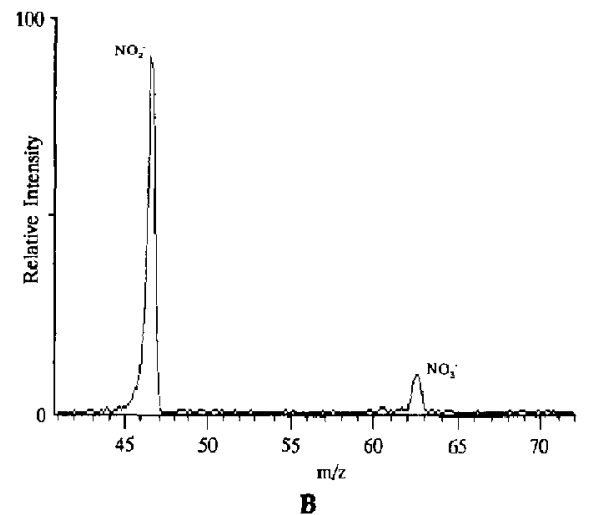

Figure 8. Demonstration of specificity for mass-selective ion ejection in a region of the ASGDI mass spectrum for air (a) without and (b) with the swept-frequency resonance ejection/fixed-amplitude if technique.

and 62, whereas a decrease in the resonance ejection amplitude resulted in only partial ejection of the targeled masses. Thus, there can be a trade-off between mass discrimination and ejection efficiency, although in this instance conditions existed for complete ejection without mass discrimination over the targeted mass range.

\section{Mass-Selective Isolation}

The wide mass range ejection methods demonstrated above can also be adapted for ion isolation. The PFTBA mass spectrum in Figure 9 was obtained following mass-selective ion isolation of $m / z 100-200$, previously targeted for ion ejection, using an if ramp/ fixed-frequency resonance ejection scan function: a $5-\mathrm{V}(\mathrm{p}-\mathrm{p})$ resonance ejection signal in resonance with $m / z 200$ was activated for $5 \mathrm{~ms}$ as the rf level was ramped over a range that corresponded to $m / z$ 25-100. Ions below $m / z 100$ became sequentially unstable as their $q_{z}$ values exceeded 0.908 , while concurrently ions between $m / z 200$ and $m / z 800$ were sequentially ejected as their secular frequencies became resonant with the resonance ejection frequency. Consequently, ions between $m / z$ 100-200 and ions of

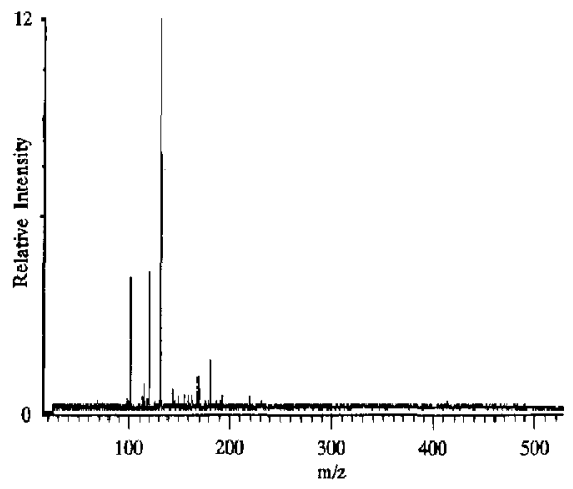

Figure 9. PFI'BA mass spectrum following mass-selective isolation of ions at $m / z 100-200$ via rf-ramp/fixed-frequency resonance ejection.

$m / z>800$ were stored in the ion trap. The sweptfrequency resonance ejection method was also used for mass-selective isolation of the same mass range by using a two-stage scan function: the mass-selective instability method was used to eject ions less than $m / z 100$ by ramping the rf voltage to a level corresponding to $m / z 100$, then the resonance ejection frequency was swept over the frequency range 15-28 $\mathrm{kHz}$, causing ions above $m / z 200$ to be removed via resonance ejection. Alternatively, the two mass regions could be ejected by sweeping the resonance ejection frequency over two different ranges at a fixed If voltage or by sweeping over a single frequency range at two distinct rf levels.

Besides being used for isolation of ions throughout a continuous mass range, these methods can also be adapted for concurrent isolation of ions having multiple, widely spaced $m / z$ values while excluding those at intermediate masses. Figure 10 shows a PFTBA mass spectrum in which ions at $m / z 100$ and 219 were isolated by using the following scan function. The operational sequence for isolation began by raising the if amplitude so that ions below $m / z 100$ were ejected. After the rf amplitude was returned to the

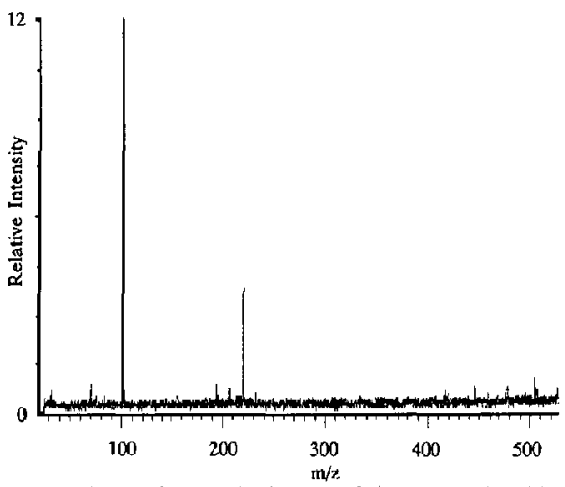

Figure 10. Mass-selective isolation of ions at $m / z 100$ and 219 in the PFTBA mass spectrum via swept-frequency resonance ejection at sequential, stepped $\mathrm{rf}$ amplitudes. 
initial level, ions in the range between $m / z 100$ and 219 were next removed via swept-frequency resonance ejection. In the final ejection step, the rf potential was raised prior to another frequency sweep so that ions over the $m / z$ range $219-490$ were brought into resonance over the same resonance ejection frequency range used in the previous step. Thus, ions at $m / z 100$ and 219 were simultaneously isolated in the ion trap. The scan function was limited to a single frequency range because the function generator sweep limits were set manually. Obviously, the same result could be achieved without changing the amplitude of the fundamental radio frequency by applying a sequence of resonance ejection stages over separate frequency ranges.

These results indicate that the procedures used for ion ejection as depicted in Figures 2 and 3 can also be adapted for isolation of ions. It must be emphasized that with these wide-mass-range isolation methods any ions present with mass-to-charge ratios greater than an upper $m / z$ limit will also remain in the ion trap. Inspection of Figures 2 and 3 reveals that this upper $m / z$ limit is determined by the if amplitude and resonance ejection frequency parameters used in the scan function. Therefore, judicious use of these techniques requires awareness of the $m / z$ limits imposed by the relevant parameters in the isolation scan function. In the previously mentioned mass-selective storage techniques employing combined application of $\mathrm{rf}$ and dc voltages, only those ions having $a_{z}$ and $q_{z}$ values on the working line and within the stability region boundaries are stored in the ion trap. The mass range isolated by the rf-dc method is determined by the slope ( $U / V$ ratio) of the working line and hence by its points of intersection with the boundaries $\left(\beta_{z}=\right.$ 0 and $\left.\beta_{z}=1\right)$ of the stability region. Therefore, all other low- and high-mass ions are excluded from the trapping region. However, ions at multiple, discrete $m / z$ values cannot be simultaneously isolated using the rf-dc method.

\section{Analytical Applications}

The previous examples involved ion isolation after ion accumulation. In situations where ion accumulation time is limited due to the rapid buildup of matrix ions, ion ejection methods are potentially most useful when applied during ion accumulation. This can be done using the combined rf-dc method for ion isolation when forming ions by EI within the ion trap, that is, the usual method for forming ions in a quadrupole ion trap. There are a growing number of applications, however, where concurrent ion accumulation $/ \mathbf{r f}$-dc isolation is not effective. Two important applications are immediately apparent. These are chemical ionization $(\mathrm{CI})$ in the ion trap and ion injection into the ion trap. In $\mathrm{CI}$, typically a reagent ion with a low $\mathrm{m} / \mathrm{z}$ value must be trapped along with the analyte ions produced during the ionization period. The rf-dc method cannot be used to store ions of two widely disparate $\mathrm{m} / \mathrm{z}$ values while rejecting ions of intermediate $m / z$ values. In ion injection, it has been demonstrated that injection efficiency for ions of a particular $\mathrm{m} / \mathrm{z}$ value is typically optimized at fundamental $\mathrm{rf}$ amplitudes well below those used for $\mathrm{rf} / \mathrm{dc}$ isolation of ions of the same mass-to-charge ratio formed within the ion trap (21-23). This is particularly true for ions with mass-to-charge ratios greater than 100 . Alternative approaches to mass selective rejection of ions during the ion accumulation period are therefore desirable for $\mathrm{CI}$ and for ion injection.

The ability to accumulate ions selectively is useful in increasing the dynamic range of the ion trap when the ions of interest constitute a small fraction of the total ion signal. It is important to distinguish this situation from those where other approaches to extending the dynamic range of the ion trap are used. For example, automatic gain control (ACC) [24] is a technique developed to increase the ion trap dynamic range by varying ionization time. Although the total number of ions created by using AGC is varied by the ionization time, the relative abundance of the ions remains constant. That is, AGC does not enrich some ions by discriminating against others. Therefore, AGC is most effective when the analyte ions are the most abundant ions in the spectrum, as is usually the case when the mass spectrometer is coupled with a separator, such as a chromatograph. However, in cases where the analyte ions constitute only a small fraction of the ions formed within or injected into the ion trap. dynamic range is primarily limited by space charge due to the matrix ions. It is in these instances where enriching the ions of interest, as shown here via resonance ejection, allows extension of dynamic range by increasing ion accumulation time. In the TNT detection example given here, $\Lambda C C$ alone would not allow for significant dynamic range extension due to space charge from the lower mass ions. However, ACC would be effective in concert with resonance ejection to reduce or eliminate the accumulation of space charge due to nonanalyte ions.

Experiments with both single-frequency resonance ejection/rf ramp and swept-frequency resonance ejection/fixed if approaches to ion rejection were performed to investigate their utility for reducing space charge due to unwanted matrix ions. The experiment involving the injection of anions formed from TNT in the ASGDI source is an excellent illustrative case as the TNT-related anions constitute less than 1\% of the ions in the mass spectrum. In the ion injection case, a swept-frequency/fixed $\mathrm{rf}$ approach is clearly preferable to a single-frequency/rf ramp approach: it is desirable to maximize injection efficiency by fixing the amplitude of the fundamental if and to reject unwanted ions by sweeping the resonance ejection frequency or by applying a relatively large amplitude (30 $\mathrm{V}$ p-p) fixed resonance ejection frequency. We have found the latter approach to be effective in rejecting 


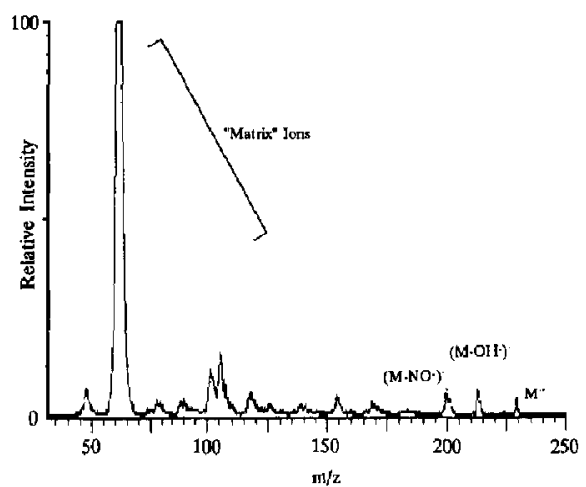

A

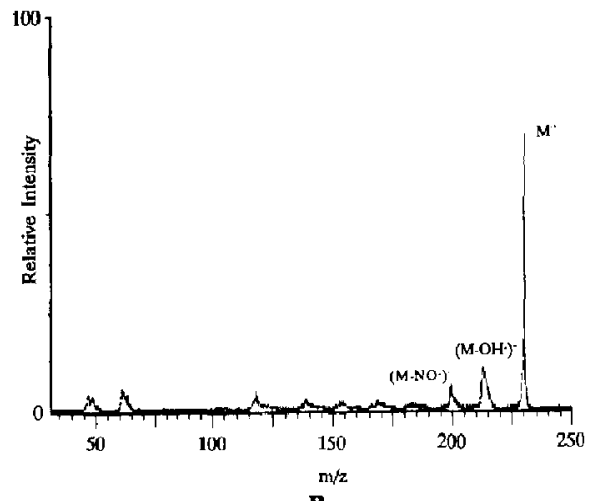

B

Figure 11. Mass spectra for 1.5-s injection of anions from air-sustained glow discharge ionization of TNT (a) without and (b) with concurrent ejection of ions at $\mathrm{m} / \mathrm{z} 46-120$ via the swept-frequency resonance ejection/ficed-amplitude if technique.

ions over a mass-to-charge range several tens of $\mathrm{m} / \mathrm{z}$ units wide and centered around a mass-to-charge having a fundamental secular frequency equal to the resonance ejection frequency. This approach is less selective than sweeping the resonance ejection frcquency but is simpler and just as effective for cases in which most of the unwanted ions fall within a massto-charge range of about 50 . It was found that ramping the fundamental rf amplitude during ion injection, on the other hand, gave poorer TNT-related signals than the fixed $\mathrm{rf}$ approaches for the same ion injection time. This was due to the fact that ion injection efficiency for the analyte anions of interest was not constant over the fundamental rf amplitude range necessary to remove the matrix anions. This application is therefore illustrated here using the fixed If approaches to ion isolation.

Figure 11a shows the mass spectrum obtained under the same conditions as those for the mass spectrum of Figure $6 \mathrm{~b}$ except that the injection time was $1.5 \mathrm{~s}$ as opposed to $10 \mathrm{~ms}$. Several noteworthy observations can be made in comparing Figure 11a with Figure $6 \mathrm{~b}$. For example, no clearly deleterious effects due to space charge are apparent at the short injection time, whereas the peaks are noticeably broadened at the longer injection time, as is commonly observed when long ion accumulation times are used. The furst indication of space charge is usually a broadening of the peaks in the mass spectrum, particularly at low $m / z$ values. This is due to the fact that ions are ejected from the trap from low $m / z$ to high $m / z$ so that the total number of ions in the ion trap (and hence, space charge) decreases steadily as the scan of the fundamental if amplitude proceeds. Second, the total ion signal obtained under the conditions used to acquire Figure 11a is far from 150 times that observed in Figure $6 \mathrm{~b}$, although the ion accumulation time is 150 times longer. (A quantitative comparison cannot be made with these figures, because Figure 11a is not normalized. However, both were acquired at the same sensitivity, and the normalized data (not shown) indicate that the total ion signals obtained from the two experiments are almost the same.) This observation reflects the finite ion-storage capacity of the ion trap. It is also striking that the signals at $m / z 46$ and $m / z$ 48 constitute the base peaks in Figure $6 \mathrm{~b}$ but are relatively small in Figure 11a. This reflects the fact that, at any given set of $a_{u}$ and $q_{u}$ conditions, the degree to which space charge affects trapping and, perhaps, injection efficiency is mass-dependent. It is beyond the scope of this discussion to address these issues in detail. Space-charge effects have been addressed by several groups [14, 25, 26]. These works have shown that the ion population can significantly alter the boundaries of the stability diagram such that ions of some mass-to-charge ratios that might otherwise be trapped become unstable when space charge becomes significant. We are currently looking into this situation in detail. Qualitatively, we observe that when space charge becomes significant, ions in both high and low extremes of the $m / z$ range that would ordinarily be trapped are discriminated against more strongly than the ions of intermediate $m / z$ values.

The effects of spacc charge in situations in which ions of widely different $\mathrm{m} / \mathrm{z}$ values are present are not well understood, particularly when ion injection is used to accurnulate the ions. Empirically, we see that space charge limits dynamic range and introduces mass discrimination. Some form of resonance ejection during ion accumulation can be useful in this regard. Figure $11 \mathrm{~b}$ shows the mass spectrum acquired under the same conditions as those used to acquire the spectrum of Figure 11a except that the swept-frequency resonance ejection/fixed if technique was used to scan out ions from $m / z 46-120$ continually during ion accumulation. The low-mass ions that appear in the spectrum are those that were admitted near the end of the ion injection period and after the final ramp of resonance ejection frequency passed the frequencies necessary to eject them.

Comparison of Figure 11a and b suggests that signals due to TNT-derived ions can be increased simply by increasing the ion injection time provided the much 


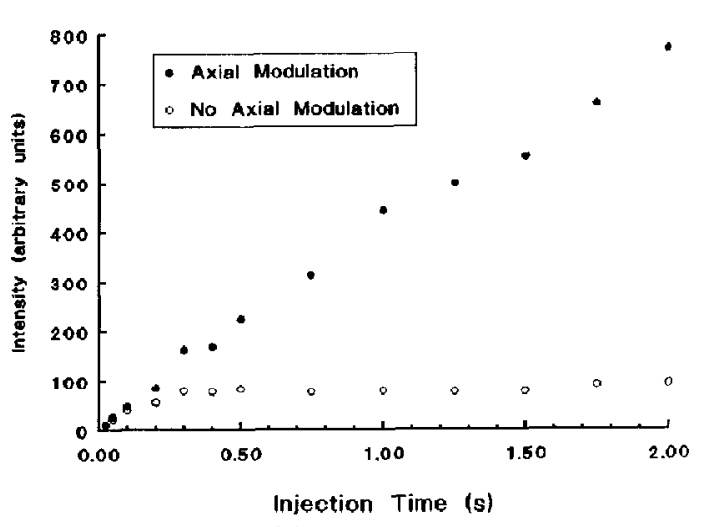

Figure 12. Extension of linear dynamic range via resonance ejection for plot of total TNT-related ion $(m / z 197,210,227)$ intensity versus ion injection time.

more abundant matrix ions are not permitted to accumulate. This is clearly demonstrated in Figure 12, which shows plots of the total TNT-derived ion signal (i.e., the sum of the peak areas due to the ions at $\mathrm{m} / \mathrm{z}$ 197,210 , and 227) as a function of ion injection time with and without the use of resonance ejection. In this case, a single resonance ejection frequency was applied, corresponding to the secular frequency of an ion at $\mathrm{m} / \mathrm{z} 55$, at an amplitude of $30 \mathrm{~V}$ p-p. This effectively prevented the accumulation of ions below $m / z 100$. As the figure demonstrates, the total TNTderived signal increases linearly with injection time out to an ion accumulation time of $2.0 \mathrm{~s}$ when resonance ejection is used to prevent the much more intense low-mass ions from accumulating. Without resonance ejection, however, deviation from linearity is observed at injection times as short as about $\mathbf{5 0}$ ms. The total signal quickly levels off beyond $0.2 \mathrm{~s}$ injection and remains essentially constant out to $2.0 \mathrm{~s}$ injection. At this time, almost all of the TNT-related signal is found in the lowest mass ion (not shown), $m / z$ 197. This is consistent with our general observation that space charge tends to introduce mass discrimination against high-mass ions (see above). The point at which this effect is observed for any particular ion depends, of course, on initial $a_{u}$ and $q_{u}$ conditions as well as on the charge density.

Ion ejection via resonance ejection is also finding an important role in the analysis of ions with massto-charge ratios greater than the nominal mass range of the ion trap. Kaiser et al. [11] and McLuckey et al. [27] have used similar resonance ejection techniques to isolate ions of $\mathrm{m} / \mathrm{z}$ valucs greater than 650 (the nominal mass range of the ITMS) for MS/MS studies. In such a case, the necessary if and dc voltages required to isolate the ions of interest are not available from the instrument electronics. Nevertheless, the ions can be isolated using either fixedfrequency/swept-rf or swept-frequency/fixed-rf forms of resonance ejection. This is illustrated with the former approach for ions formed by electrospray from

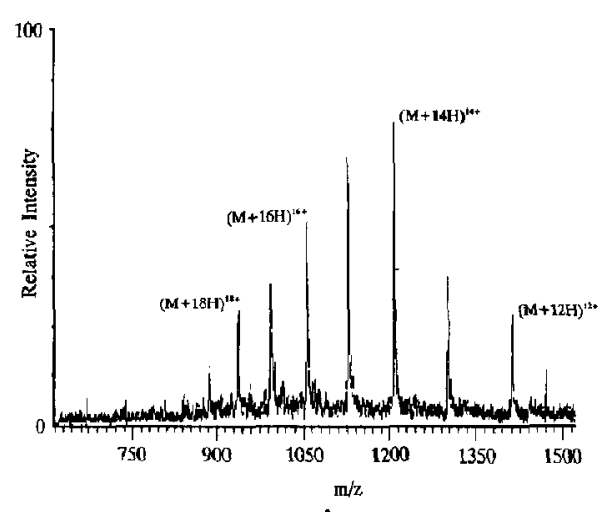

A

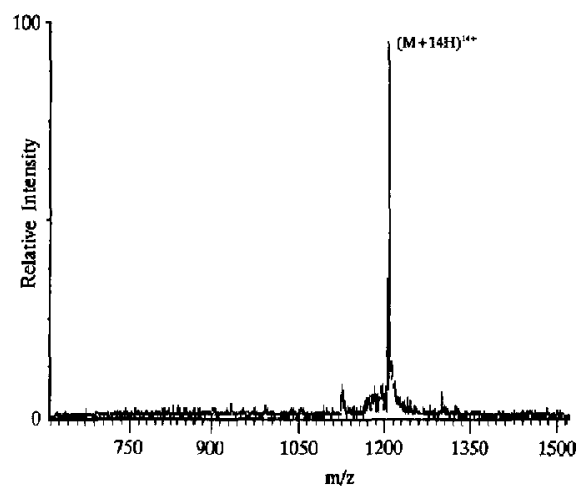

B

Figure 13. Electrospray ionization mass spectra for myoglobin (average MW 16,950.4) (a) before and (b) after isolation of the $[\mathrm{M}+14 \mathrm{H}]^{14+}$ ion using resonance ejection. Resonance ejection was also used to extend the mass range of the ion trap by a factor of 3 .

the protein myoglobin (average $\mathrm{MW}=16,950.4$ ). Figure 13 shows the electrospray mass spectrum for myoglobin before (Figure 13a) and after (Figure 13b) resonance ejection was used to isolate the $[M+$ $14 \mathrm{H}]^{14+}$ ion. (In both cases resonance ejection was also used during the final ramp of the fundamental if amplitude to extend the mass range of the ion trap by a factor of 3. This, of course, is another important application of resonance ejection, as mentioned above.) The $[\mathrm{M}+14 \mathrm{H}]^{14+}$ charge state was isolated after ion injection in two steps. The first step involved ramping the rf amplitude from 577 to $1154 \mathrm{~V}(0-\mathrm{p})$ while applying a $7-\mathrm{V}(\mathrm{p}-\mathrm{p}), 14.5-\mathrm{kHz}$ sine wave to the endcaps to remove the lower charge states (higher $m / z)$. The second step involved ramping the rf amplitude from 4038 to $7500 \mathrm{~V}(0-\mathrm{p})$ while applying a $17-\mathrm{V}$ (p-p), 200.5-kHz sine wave to the endcaps to remove the higher charge states (lower $m / z$ ). The slightly greater signal due to $[M+14 \mathrm{H}]^{14+}$ in Figure $13 \mathrm{~b}$ falls within experimental reproducibility. Note that small signals due to the +15 and +13 charge states are still evident after the two resonance ejection steps. These ions could be removed more effectively either by applying a greater amplitude sine wave to the end 
caps, at the risk of ejecting some of the ions of interest, or by scanning the if amplitude more slowly to give the ions more time to absorb power from the endcaps. Unfortunately, the ion trap hardware does not readily permit the amplitude of the fundamental If to be scanned more slowly than was done here. In principle, however, complete removal of the other charge states is possible. One way to accomplish this is to use the swept-frequency/fixed-rf approach with two relatively slow sweeps of the resonance ejection frequency either over two distinct frequency ranges at a constant if amplitude or with the same frequency range but at two distinct rf amplitudes.

\section{Summary}

Two approaches for mass-selective ion manipulation over a wide mass range have been used in these resonance ejection studies. The first involves applying a single, fxed frequency to the endcaps and scanning the amplitude of the fundamental rf to bring ions into resonance with the resonance ejection frequency. This is somewhat analogous to the resonance power absorption approach once used in ion cyclotron resonance (ICR) spectroscopy whereby a discrete frequency was applied to two apposite plates of the ICR cell and the magnetic field was scanned to bring ions into resonance with the applied frequency. The second approach involves scanning the resonance ejection frequency at a fixed rf amplitude. This is somewhat analogous to the frequency sweep approach used in ICR spectroscopy whereby the magnetic field is fixed and the frequency applied to the excitation plate is varied. It is important to note that the former approach can be used with an unmodified version of the commercially available ITMS. Although the latter approach currently requires additional hardware to allow for scanning the resonance ejection frequency, use of the technique would become straightforward by commercial integration of a programable sweep generator and requisite software into the ITMS. Nevertheless, both approaches constitute powerful and flexible means for ion isolation in the quadrupole ion trap that go beyond the isolation of ions of a single $m / z$ value or small $m / z$ range as afforded by the combined rf-dc approach.

\section{Acknowledgments}

This research was sponsored by the U.S. Department of Energy Office of Safeguards and Security under contract DE-AC05840R21400 with Martin Marietta Energy Systems, Inc.

\section{References}

1. Stafford, G. C.; Kelley, P. E.; Syka, J. E. P.; Reynolds, W. E.; Todd, J. F. J. Int. J. Mass Spectrom. Ion Processes 1984, 60, 85.
2. Stafford, G. C.; Kelley, P. E.; Stephens, D. R. U.S. Patent $4,540,884,1985$.

3. Kelley, P. E.; Stafford, G. C.; Fies, W. J.; Syka, J. E. P.; McIver, R. T.; Hunter, R. L.; Todd, J. F. J.; Bexon, J. J. Praceedings of the 32nd Annual Conference on Mass Spectrometry and Allied Topics, San Antonio, 1984; $p 505$.

4. Dawson, P. H.; Whetten, N. R. AFCRL-69-0185, 1969.

5. Fulford, J. E.; March, R. E. Int. J. Mass Spectrom. Ion Phys. 1978, 26, 155 .

6. Louris, J. N.; Cooks, R. G.; Syka, J. E. P.; Kelley, P. E.; Stafford, G. C.; Todd, J. F. J. Anal. Chem. 1987, 59, 1677.

7. Louris, J. N.; Brodbelt-Lustig, J. S.; Cooks, R. G.; Glish, G. L.; Van Berkel, G. J.; McLuckey, S. A. Int. J. Mass Spectrom. Ion Processes 1990, 94, 15.

8. McLuckey, S. A.; Glish, G. L.; Kelley, P. E. Anal. Chem. $1987,59,1670$.

9. Tucker, D. B.; Hameister, C. H.; Bradshaw, S. C.; Hoekman, D. J.; Weber-Grabau, M. Proceedings of the 36th ASMS Conference on Mass Spectrometry and Allied Topics, San Francisco, 1988; p 628.

10. Kaiser, R. E., Jr.; Cooks, R. G.; Moss, J.; Hemberger, P. H. Rapid Commun. Mass Spectrom. 1989, 3, 50.

11. Kaiser, R. E.; Louris, J. N.; Amy, J. W.; Cooks, R. G. Rapid Commun. Mass Spectrom. 1989, 3, 225.

12. Van Berkel, G. J.; Glish, G. L.; McLuckey, S. A. Anal. Chem. 1990, 62, 1284 .

13. March, R. E.; Hughes, R. J.; Quadrupole Storage Mass Spectrometry; Winefordner, J. D.; Kolthoff, I. M., Eds.; Chemical Analysis Series 102; Wiley: New York, 1989.

14. Fischer, E. Z. Phys. 1959, 156, 1.

15. Fulford, J. E.; Hoa, D.-N.; Hughes, R. J.; March, R. E.; Bonner, R. F.; Wong, G. J. J. Vac. Sci. Technol. 1980, 17, 829.

16. Todd, J. F, J.; Bexon, J. J.; Smith, R. D; Weber-Grabau, M.; Kelley, P. E.; Syka, J. E. P.; Stafford, G. C.; Bradshaw, S. C. Proceedings 16th Meeting British Mass Spectrometry Society, York, U.K., 1987; p 206.

17. Kelley, P. E.; Syka, J. E. P.; Ceja, P. C.; Stafford, G. C.; Louris, J. N.; Grutzmacher, H. F.; Kuck, D.; Todd, J. F. J. Proceedings of the 34th Conference on Mass Spectrometry and Allied Topics, Cincinnati, 1986; $\mathrm{p} 963$.

18. Syka, J. E. P.; Fies, W. J., Jr. Proceedings of the 35th Conference on Mass Spectrometry and Alied Topics, Denver, 1987; $\mathrm{p}$ 767.

19. McLuckey, S. A.; Glish, G. L.; Asano, K. G. Anal. Chim. Acta 1989, 225, 25.

20. McLuckey, S. A.; Glish, G. L.; Asano, K. G.; Grant, B. C. Anal. Chem. 1988, 60, 2220.

21. Louris, J, N.; Amy, J. W.; Ridley, T. Y.; Cooks, R. G. Int. J. Mass Spectrom. Ion Processes 1989, 88, 97.

22. Pedder, R. E.; Yost, R. A.; Weber-Grabau, M. Proceedings of the 37th ASMS Conference on Mass Spectrometry and Allied Topics, Miami Beach, 1989; p 468.

23. Schwartz, J. C.; Cooks, R. G.; Weber-Grabau, M.; Kelley, P. E. Proceedings of the 36 th ASMS Conference on Mass Spectrometry and Allied Topics, San Francisco, 1988; $\mathrm{p} 634$.

24. Stafford, G. C.; Taylor, D. M.; Bradshaw, S. C.; Syka, J. E. P.; Uhrich, M. Proceedings of the 35th Conference on Mass Spectrometry and Allied Topics, Denver, 1987: p 775.

25. Todd, J. F. J.; Waldren, R. M.; Mather, R. E. Int. I. Mass Spectrom. Ion Phys. 1980, 34, 325.

26. Schwebel, C.; Moller, P. A.; Manh, P.-T. Reo. Phys. Appl. 1975, 10, 227.

27. McLuckey, S. A.; Van Berkel, G. J.; Glish, G. L. I. Am. Chem. Soc. 1990, 112, 5668 . 